\title{
Hospital satisfaction does not predict functional outcome one year after total shoulder arthroplasty
}

\author{
Robert D. Wojahn ${ }^{1}$, Joan M. Atencio ${ }^{1}$, Julianne A. Sefko ${ }^{1}$, Leesa M. Galatz ${ }^{2}$, Jay D. Keener ${ }^{1}$, \\ Ken Yamaguchi ${ }^{1}$, Aaron M. Chamberlain ${ }^{1 \wedge}$
}

${ }^{1}$ Department of Orthopedic Surgery, Washington University, St. Louis, MO, USA; ${ }^{2}$ Mount Sinai Mount Sinai Health System, New York, NY, USA Contributions: (I) Conception and design: RD Wojahn, K Yamaguchi, AM Chamberlain; (II) Administrative support: JM Atencio, JA Sefko; (III) Provision of study materials or patients: LM Galatz, JD Keener, K Yamaguchi, AM Chamberlain; (IV) Collection and assembly of data: RD Wojahn, JA Sefko, JM Atencio, AM Chamberlain; (V) Data analysis and interpretation: RD Wojahn, AM Chamberlain; (VI) Manuscript writing: All authors; (VII) Final approval of manuscript: All authors.

Correspondence to: Aaron M. Chamberlain, MD, MSc, MBA. Associate Professor, Shoulder \& Elbow Service, Washington University Orthopedics, Barnes Jewish Hospital, 425 S. Euclid Ave., Campus Box 8233, St. Louis, MO 63110, USA. Email: amchamberlain@wustl.edu.

Background: Healthcare is shifting to value-based payment models. Two percent of Medicare reimbursements are currently linked to value measures including the Hospital Consumer Assessment of Healthcare Providers and Systems (HCAHPS) hospital satisfaction survey. The purpose of this study was to determine if HCAHPS survey results are correlated with validated legacy outcome measures after total shoulder arthroplasty.

Methods: A prospective observational study was conducted in 84 patients undergoing elective total shoulder arthroplasty. Baseline 12-item Short-Form Health Survey (SF-12), American Shoulder and Elbow Surgeons (ASES), and Western Ontario Osteoarthritis of the Shoulder Index (WOOS) questionnaires were completed at the time of enrollment. ASES and WOOS scores were collected at 3-month and 1-year postoperatively. Patients were contacted to complete the HCAHPS survey postoperatively. HCAHPS results and baseline functional scores were evaluated for an association with improvements in legacy outcome measures after surgery.

Results: HCAHPS scores were higher among males than females $(\mathrm{P}=0.04)$. Age, SF-12 physical component scores, SF-12 mental component scores, and pre-operative symptom severity were not associated with HCAHPS results. HCAHPS scores were not correlated with ASES ( $r=0.09, \mathrm{P}=0.44)$ or WOOS scores $(\mathrm{r}=-0.17, \mathrm{P}=0.13)$ at one year after surgery. HCAHPS was also not correlated with the absolute improvement in ASES $(\mathrm{r}=-0.02, \mathrm{P}=0.85)$ or WOOS scores $(\mathrm{r}=-0.08, \mathrm{P}=0.48)$ from pre- to one year post-operatively.

Conclusions: The HCAHPS score, a measure of satisfaction and a determinant of Medicare quality-based reimbursement, showed no correlation with functional outcome measures at one year after total shoulder arthroplasty. Thus, HCAHPS patient satisfaction survey may not be aligned with functional outcomes valued by patients. Further consideration is warranted regarding the assessment of quality, and in turn reimbursements, with survey results.

Keywords: Patient outcomes; orthopedic surgery; shoulder replacement; Hospital Consumer Assessment of Healthcare Providers and Systems (HCAHPS)

Received: 08 May 2020; Accepted: 15 February 2021; Published: 25 December 2021.

doi: $10.21037 /$ jhmhp-20-51

View this article at: http://dx.doi.org/10.21037/jhmhp-20-51

\footnotetext{
^ ORCID: 0000-0002-4622-9797.
} 


\section{Introduction}

With a significant focus on quality and patient satisfaction, health care reimbursement has been transitioning from the traditional fee-for-service model to value-based payment. This is underscored by the establishment of the Hospital Value-Based Purchasing program in 2010 which now links $2 \%$ of Medicare reimbursements to four hospital quality of care domains: Clinical Care, Safety, Efficiency and Cost Reduction, and Patient- and Caregiver-Centered Experience of Care/Care Coordination (1,2). While the first three domains consider clinical metrics such as mortality rates for common illnesses, complication rates, and costs of care, the experience of care domain is calculated exclusively from the Hospital Consumer Assessment of Healthcare Providers and Systems (HCAHPS) survey. This survey was developed by the Centers for Medicare \& Medicaid Services (CMS) in 2008 as a standardized method to evaluate and compare patient satisfaction among US hospitals. Per protocol, it is administered to a random sample of inpatients between 48 hours and 6 weeks after discharge and assesses topics such as physician and nurse communication, room cleanliness, pain control, and discharge education $(2,3)$.

It has been concluded in non-orthopaedic literature that HCAHPS scores correlate with clinical outcomes and therefore serve as a useful proxy for quality of care under a value-based model (4-8). In one study, Stein et al reported that patient experience as reported by HCAHPS surveys correlated with the quality of care in terms of having an inverse relationship with complication rates (6). In another study, Tsai et all evaluated HCAHPS survey data among surgical patients and found that patient outcomes metrics such as shorter length of hospital stay, lower readmission rates and lower mortality were significantly better in hospitals with HCAHPS scores in the highest quartile $v s$. those in the lowest quartile (7). However, relatively little is known about the relationship of HCAHPS survey scores and validated outcomes measure in the orthopaedic surgery population. Two recent studies considering the overall hospital satisfaction component of HCAHPS found no association between this rating and validated functional outcome measures after lumbar spine surgery or total hip arthroplasty $(9,10)$.

Legacy outcome measures are routinely utilized to quantify patient function, pain, and activity level and thus serve as useful quality measures after orthopaedic surgery. The American Shoulder and Elbow Surgeons (ASES) score was developed in 1994 as a general shoulder outcome tool and evaluates patients' pain, instability, and activities of daily living (11). The Western Ontario Osteoarthritis of the Shoulder Index (WOOS) score was created in 2001 to be the primary outcome measure in clinical trials studying patients with osteoarthritis of the shoulder (9). Both of these outcome measures have been validated by the orthopaedic community and are widely accepted as markers of shoulder function for evaluation and comparison of treatments (12-14). The 12-item Short-Form Health Survey (SF-12) has been shown to be a psychometrically sound tool in assessing the health-related quality of life in patients with osteoarthritis (15). The HCAHPS survey was developed by the Center for Medicare and Medicaid Services (CMS) in partnership with the Agency for Healthcare Research and Quality (AHRQ) and after being found psychometrically acceptable, was implemented as a patient hospital experience survey and is public domain (16).

The primary aim of this study was to determine if the Hospital Consumer Assessment of Healthcare Providers and Systems survey (HCAHPS) patient satisfaction scores, an increasingly utilized metric in today's healthcare environment, correlate with improvements in legacy functional outcome measures after total shoulder arthroplasty. We hypothesized that no correlation would exist between these measures, indicating that HCAHPS is not useful as a proxy for quality of care after shoulder arthroplasty. Secondary aims were to determine if a relationship exists between HCAHPS and patient age, gender, or general health status as assessed by the SF-12 questionnaire (17).

We present the following article in accordance with the SURGE reporting checklist (available at http://dx.doi. org/10.21037/jhmhp-20-51).

\section{Methods}

\section{Patients}

The study was conducted in accordance with the Declaration of Helsinki (as revised in 2013). The study was approved by the Washington University in St. Louis Institutional Review Board (IRB \#: 201410148) and informed consent was taken from all patients. A prospective observational study was conducted including patients undergoing elective primary anatomic total shoulder arthroplasty by one of 4 fellowship trained shoulder surgeons to manage osteoarthritis at a single institution tertiary referral center in the Midwestern United States. Exclusion criteria included age $<55$ or $>81$ 
for whom the legacy outcome measures are not validated, revision procedures, and inability to complete at least one year of mail-in or electronic follow-up. Consecutive patients were approached by a member of the study team (RDW) and provided consent for study enrollment prior to or at the time of surgery from March 2015 to December 2016. Patients undergoing staged bilateral total shoulder arthroplasty were only enrolled for a single side to ensure all participants represented independent cases.

\section{Measures and outcomes}

Participants completed the ASES and WOOS questionnaires at the time of enrollment detailing their pre-operative symptoms. They also completed the SF-12 general health questionnaire.

Per CMS guidelines, patients were contacted by a thirdparty survey company between 48 hours and 6 weeks after hospital discharge to complete the HCAHPS survey by telephone. All patients enrolled in this study were attempted to be contacted one time by the survey company to complete the HCAHPS survey. Table 1 displays the individual questions that make up the HCAHPS survey.

Patients completed the ASES and WOOS questionnaires by mail or online at the 3-month and 1-year post-operative time points. They were also asked to complete a selfreported satisfaction questionnaire about how effective surgery was in improving pain and function (0-10 ordinal scale). Patients who failed to complete the enrollment packet, HCAHPS survey, or the 1-year ASES followup survey were excluded from the analysis. No incentives (financial or other) were provided to study participants.

\section{Procedures}

No modifications were made of surgeons' intra-operative or post-operative protocols. In addition to general anesthesia, all patients received an interscalene nerve block with placement of an indwelling nerve catheter that remained in place for two to three days postoperatively. After surgery, patients were admitted to a surgical floor with nurses who exclusively care for post-operative orthopaedic patients. Patients were seen by an occupational therapist on the first post-operative day for rehabilitation instruction and education. This included early active assistive range motion with pulleys and/or a continuous passive range of motion machine. Cryotherapy was utilized in all patients. Patients were discharged to home or a skilled nursing facility based on the recommendation of therapy services.

\section{Statistical analysis}

Pre-enrollment power analysis demonstrated that 47 patients were necessary to detect a correlation with an effect size of $r=0.4(\beta=0.8, \alpha=0.05)$ between HCAHPS scores and improvements in ASES or WOOS scores.

Data were examined with descriptive statistics to determine frequencies for categorical variables and means for continuous variables. HCAHPS survey results were converted to a numerical score using previously published methodology (18). ASES, WOOS and SF-12 responses were tallied which provided the respective score. The correlation between HCAHPS and legacy outcome measures was explored using Spearman correlation analyses given the non-normal distribution of HCAHPS results. The MannWhitney U, Wilcoxon signed rank, Pearson, and Spearman correlation tests were used to examine associations between age, gender, SF-12 results, HCAHPS, and legacy outcome measures, as appropriate for their distributions. Patients with missing data were excluded from analyses involving the missing variable. All statistical analysis was performed using SPSS Statistics software (IBM).

\section{Results}

Approximately 200 patients were approached for study enrollment of which 163 consented to participation. Of these participants, 18 failed to complete the enrollment packet, 57 did not complete the HCAHPS survey due to not answering the phone call from the survey company administering the HCAHPS survey after 3 attempts, 3 patients failed to complete the 1-year follow-up questionnaires, and 1 patient died before completing 1 year of follow-up. The remaining 84 patients were included in the study. Eighty-two of these patients had 1-year postoperative ASES data and the remaining two had 2-year post-operative data that was substituted for the missing 1-year data. Seventy-nine patients had 1-year post-operative WOOS data.

Of the 84 included patients, forty-six patients (55\%) were male and the average age was 66.3 years (range, 55.480.8 years). Baseline SF-12 physical component scores averaged 32.3 (range, 15.6-51.2) and mental component scores averaged 49.0 (range, 18.3-67.8). Pre-operative, 3 -month post-operative, and 1-year post-operative ASES and WOOS scores are summarized in Table 2. Absolute 
Table 1 HCAHPS survey questions

During this hospital stay, how often did nurses treat (you/your family member) with courtesy and respect?

During this hospital stay, how often did nurses listen carefully to (you/your family member)?

During this hospital stay, how often did nurses explain things in a way (you/your family member) could understand?

During this hospital stay, after (you/your family member) pressed the call button, how often did (you/your family member) get help as soon as (you/he or she) wanted it?

During this hospital stay, how often did doctors treat (you/your family member) with courtesy and respect?

During this hospital stay, how often did doctors listen carefully to (you/your family member)?

During this hospital stay, how often did doctors explain things in a way (you/your family member) could understand?

During this hospital stay, how often were (your/your family member's) room and bathroom kept clean?

During this hospital stay, how often was the area around (your/your family member's) room quiet at night?

How often did (you/your family member) get help in getting to the bathroom or in using a bedpan as soon as (you/he or she) wanted?

During this hospital stay, how often was (your/your family member's) pain well controlled?

During this hospital stay, how often did the hospital staff do everything they could to help (you/your family member) with (your/his or her) pain?

Before giving (you/your family member) any new medicine, how often did hospital staff tell (you/your family member) what the medicine was for?

Before giving (you/your family member) any new medicine, how often did hospital staff describe possible side effects in a way (you/he or she) could understand?

During this hospital stay, did doctors, nurses, or other hospital staff talk with (you/your family member) about whether (you/he or she) would have the help (you/your family member) needed when (you/he or she) left the hospital?

During this hospital stay, did (you/your family member) get information in writing about what symptoms or health problems to look out for after (you/your family member) left the hospital?

We want to know your overall rating of your stay at the hospital. Using any number from 0 to 10 , where " 0 " is the "Worst Hospital Possible" and "10" is the "Best Hospital Possible," what number would you use to rate this hospital during your stay?

During this hospital stay, staff took my preferences and those of my family or caregiver into account in deciding what my health care needs would be when I left.

When I left the hospital, I had a good understanding of the things I was responsible for in managing my health.

When I left the hospital, I clearly understood the purpose for taking each of my medications.

HCAHPS, Hospital Consumer Assessment of Healthcare Providers and Systems.

improvements in ASES and WOOS scores from preto post-operatively are also displayed. Legacy outcomes measures were significantly improved from pre-operative values at 3 -month and 1 -year postoperative time points $(\mathrm{P}<0.001)$. Of the 57 patients excluded for not completing the HCAHPS survey, 59.6\% were male, average age was 67.2 (range, 55.9-80.1), average pre-operative ASES scores was 41.0 (range, 0-92.6), and average pre-operative WOOS score was 1,235.0 (range, 571.0-1,802.0). None of these scales were significantly different from corresponding values for the 84 patients with complete follow-up data that were included in the study.
HCAHPS scores averaged 91.3 and ranged from 57.1 to 100. HCAHPS scores were higher among males than females (mean 93.7 vs. 88.3, $\mathrm{P}=0.04)$. Age $(\mathrm{P}=0.154)$, SF-12 physical component scores $(\mathrm{P}=0.44)$, and $\mathrm{SF}-12$ mental component scores $(\mathrm{P}=0.88)$ were not associated with HCAHPS results. Severity of pre-operative symptoms, as measured by patient-reported pain severity (0-10 scale) $(\mathrm{P}=0.46)$, ASES $(\mathrm{P}=0.31)$, and WOOS $(\mathrm{P}=0.12)$ were also not predictive of HCAHPS.

HCAHPS scores were not correlated with ASES scores at three months $(\mathrm{r}=0.22, \mathrm{P}=0.06)$ or one year $(\mathrm{r}=0.09, \mathrm{P}=0.44)$ after surgery. Similarly, HCAHPS showed no association 
Table 2 Pre- and post-operative ASES and WOOS scores

\begin{tabular}{lc}
\hline Variables & Average [range] \\
\hline ASES & $35.8[0-90]$ \\
Pre-operative & $66.0[0-100]$ \\
3 months & $29.3[-43.4$ to 90$]$ \\
3-month improvement & $81.7[3.3$ to 100$]$ \\
1 year & $45.4[-29.3$ to 93.3$]$ \\
1-year improvement & \\
WOOS & $1,282.2[486$ to 1,856$]$ \\
Pre-operative & $357.1[0$ to 1,420$]$ \\
3 months & $916.2[-289.1$ to 1,707$]$ \\
3-month improvement & $249.5[0$ to 1,353$]$ \\
1 year & $1,023.2[-478$ to 1,832$]$ \\
1-year improvement &
\end{tabular}

Higher ASES scores indicate greater function while higher WOOS scores indicate greater disability. ASES minimal clinically important difference (MCID) is 6.4 points. MCID for WOOS has not been described. ASES, American Shoulder and Elbow Surgeons; WOOS, Western Ontario Osteoarthritis of the Shoulder Index.

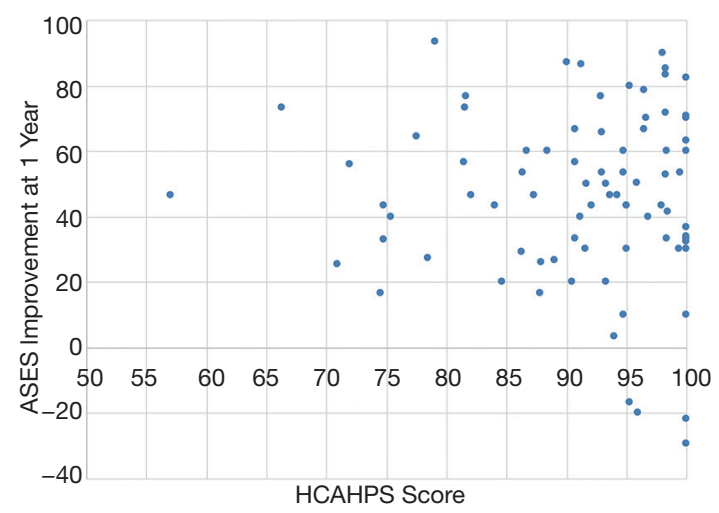

Figure 1 HCAHPS Score ASES improvement at 1 year. HCAHPS, Hospital Consumer Assessment of Healthcare Providers and Systems; ASES, American Shoulder and Elbow Surgeons.

with WOOS scores at three months $(\mathrm{r}=-0.16, \mathrm{P}=0.15)$ or one year $(\mathrm{r}=-0.17, \mathrm{P}=0.13)$ after surgery. HCAHPS was also not correlated with the absolute improvement in ASES $(\mathrm{r}=-0.02, \mathrm{P}=0.85)$ or WOOS scores $(\mathrm{r}=-0.08, \mathrm{P}=0.48)$ from pre- to one year post-operatively (Figures 1 and 2).

Women demonstrated significantly greater improvement

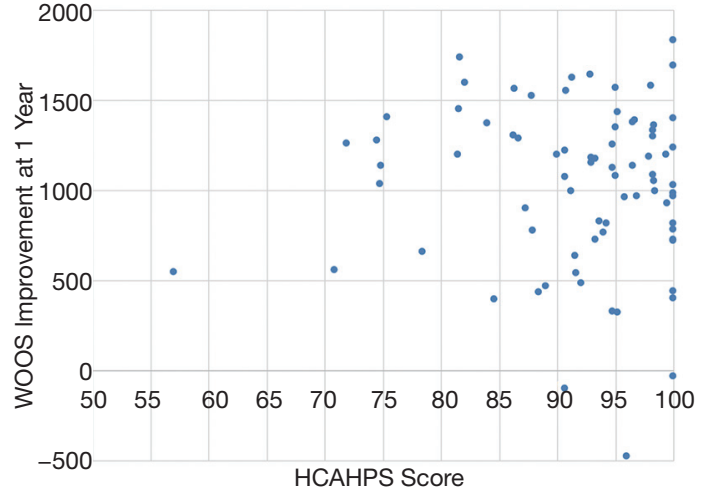

Figure 2 HCAHPS Score WOOS improvement at 1 year. HCAHPS, Hospital Consumer Assessment of Healthcare Providers and Systems; WOOS, Western Ontario Osteoarthritis of the Shoulder Index.

in ASES (mean 55.4 vs. 38.0, $\mathrm{P}=0.001$ ) and WOOS scores (1237.1 vs. 874.4, $\mathrm{P}<0.001)$ at one year after surgery. The pre-operative SF-12 physical component score, but not the mental component, was associated with ASES and WOOS improvement at one year after surgery (Table 3). Pre-operative symptom severity was also correlated with improvement in both legacy outcome measures. Age was not correlated with ASES or WOOS improvement at any time point.

The single question "How effective was the surgery in restoring a normal level of function?" at three months after surgery showed high correlation with improvements in ASES $(r=0.38, \mathrm{P}=0.001)$ and WOOS $(\mathrm{r}=0.43, \mathrm{P}<0.001)$ scores at the one-year post-operative time point. Similarly, the question "How helpful was the surgery in relieving pain?" at three month post-operatively was associated with improvements in ASES $(\mathrm{r}=0.43, \mathrm{P}<0.001)$ and WOOS $(r=0.47, P<0.001)$ scores at one year after surgery. Neither the function question $(\mathrm{r}=0.21, \mathrm{P}=0.07)$ nor the pain question $(\mathrm{r}=-0.06, \mathrm{P}=0.62)$ at three months was correlated with HCAHPS scores.

\section{Discussion}

Hospital satisfaction, as measured by the HCAHPS survey, was not correlated with improvement in legacy functional outcome measures at one year after total shoulder arthroplasty in this prospective observational study. HCAHPS was also not associated with age, baseline SF-12 scores, or pre-operative symptom severity. In contrast, patient reported improvement in pain and function at 
Table 3 Correlation of pre-operative variables and post-operative functional improvement

\begin{tabular}{lc}
\hline Variables & Correlation coefficient, $r$ (P value) \\
\hline Age & $0.21(0.06)$ \\
ASES 1 year improvement & $0.06(0.60)$ \\
WOOS 1 year improvement & \\
SF-12 PCS & $-0.24(0.03)$ \\
ASES 1 year improvement & $-0.31(0.01)$ \\
WOOS 1 year improvement & \\
SF-12 MCS & $0.01(0.93)$ \\
ASES 1 year improvement & $-0.16(0.16)$ \\
WOOS 1 year improvement & \\
Pain severity (0-10 scale) & $0.16(0.15)$ \\
ASES 1 year improvement & $0.23(0.04)$ \\
WOOS 1 year improvement & \\
Pre-Op ASES & $-0.64(<0.001)$ \\
ASES 1 year improvement & $-0.34(<0.001)$ \\
WOOS 1 year improvement & \\
Pre-op WOOS & $0.60(0.04)$ \\
ASES 1 year improvement & \\
WOOS 1 year improvement & \\
\hline ASES, American Shoulder & \\
\hline
\end{tabular}

ASES, American Shoulder and Elbow Surgeons; WOOS, Western Ontario Osteoarthritis of the Shoulder Index; PCS, physical component summary; MCS, mental component summary.

three months on a one-question survey was correlated with functional outcome measures at one year after surgery.

Strengths of this study include its prospective design and narrow, well-defined inclusion criteria including only patients undergoing an Anatomic Total Shoulder Arthroplasty performed at a single high volume institution. The findings of the present study are consistent with other studies in the orthopaedic literature showing limited or no association between HCAHPS and validated patient reported outcome measures. In a retrospective cohort study, Levin et al showed no association between HCAHPS overall hospital rating and three different outcome measures after lumbar spine surgery (10). Interestingly, they also noted higher HCAHPS scores among men than in women. Similarly, Anil and colleagues found no correlation between HCAHPS and patient-reported outcome measures after total hip arthroplasty (9). Day demonstrated no difference in patient satisfaction between patients who did and did not develop a hospital-acquired condition such as surgical site infection or venous thromboembolism after orthopaedic surgery (18). When combined with the results of this study, these results suggest HCAHPS results may not be aligned with patient priorities as related to shoulder function measured by validated patient-reported outcomes scales. Non-orthopaedic literature has also shown poor association between HCAHPS and validated outcome measures after general, vascular, transplant, urologic, and trauma surgery (19-24).

While patient experience during the hospital admission remains an important consideration in the overall care of post-arthroplasty patients, hospital satisfaction is not related to validated patient reported functional outcomes. Furthermore, many of the factors evaluated by HCAHPS, such as nursing care and room cleanliness, are out of the surgeon's control in many health care delivery models. Further consideration is warranted regarding the assessment of quality, and in turn reimbursements, with survey results. Under CMS procedures, reimbursement to hospitals is directly affected by survey results which in turn may directly or at least indirectly affect providers in health care models where providers share in hospital revenues. It is of utmost importance that hospitals and providers are aligned to provide the best possible experience and outcomes for patients. Providers need to be engaged with hospitals in decision-making and processes that affect patient satisfaction scores measured by HCAHPS.

Additionally, interpretation of patient reported outcome measures must be done with an understanding of the instruments' limits. When considering patient satisfaction, there have been numerous non-modifiable factors found to be associated with patient scores. In an investigation of the determinants of patient satisfaction after shoulder arthroplasty, Chen and colleagues found an association with age and workers' compensation status in addition to pain and functional status (25). Peres-da-Silva reported higher patient satisfaction scores among men, African Americans, and patients in the lowest quartile of socioeconomic status (26). Patient overall health status and depression have also been shown to be correlated with HCAHPS scores $(27,28)$. These studies raise concern that numerous factors outside the surgeon's control may influence patient satisfaction as measured by these instruments and in turn, perceived quality of care. Conversely, improved perioperative pain control and patient-physician communication 
have been found to be associated with higher HCAHPS scores after surgical procedures (6,28-30). Thus these may represent opportunities for improved patient satisfaction beyond excellent surgical care.

HCAHPS has been implemented by CMS and linked to reimbursement in a health care environment increasingly focused on value rather than just volume. There have been other recent studies seeking to better understand the value of care using patient satisfaction and cost data. Berglund et al. describe a method to quantify the value of shoulder arthroplasty by assigning a procedure value index after shoulder arthroplasty (31). Authors in this study reported on patient improvement as units of MCID per unit of cost of care. Using this method, greater value was reported for anatomic total shoulder arthroplasty patients compared to those undergoing reverse shoulder arthroplasty. Another study uses costs derived from a time-driven activitybased cost analysis to better understand which patient medical comorbidities are associated with a higher cost of care (32). Authors found that main drivers for cost variation an determinants of value were implant costs personnel costs. Negative patient-experience after total shoulder arthroplasty has also been assessed using patient comments and associating them with HCAHPS scores (33). This study described specific areas or aspects of care that generated a negative experience for patients. Authors found that lower HCAHPS scores correlated with patient who submitted negative comments to the hospital after their hospital stay.

There are several limitations to the present study. First, in order to optimize patient enrollment and follow-up we prospectively invited patients to participate and thus did not blind patients to study participation. Therefore, participants may have altered their survey responses to avoid disappointing their treating surgeon. We attempted to mitigate this by minimizing attending surgeon participation in study enrollment and data collection. Also, one would expect that this potential response bias would affect HCAHPS and legacy surveys to a similar extent. Next, despite enrolling 163 patients, only 84 (51.5\%) completed all necessary follow-up questionnaires. This could have introduced a selection bias if these patients differed systematically from those who did not complete appropriate follow-up but the baseline demographic data was no different between included and excluded patients. Most of the patients lost to follow-up were those who could not be reached by telephone to complete the HCAHPS survey. While this is a limitation it also represents the real sampling bias in the implementation of HCAHPS.

The HCAHPS scoring methodology utilized in this study is somewhat different than that used by CMS in the calculation of Medicare reimbursements. CMS compares hospital-wide HCAHPS data against past performance and performance of other regional hospitals. In contrast we analyzed individual patient HCAHPS data and its relationship to legacy outcome measures. This methodology has been used in numerous other studies but it is important to recognize the difference from CMS scoring.

Finally, we used ASES and WOOS scores as quality measures after shoulder surgery. One might question the use of a subjective questionnaire to assess quality rather than an objective measurement assessed by a physician, as it has been shown that subjective and objective outcome measures have variable correlation after shoulder surgery (34). For example, how should a surgeon's quality of care by judged for a patient with continued pain or limited function despite a perfectly executed surgery? This question is beyond the scope of our discussion but one that must be considered as value-based payment models becomes increasingly common.

As healthcare transitions to a value-driven model, accurate assessment of quality will be paramount. While the HCAHPS survey may offer insightful information about hospital care, it is a poor proxy for long term functional outcomes after total shoulder arthroplasty. Additional research is necessary to determine the optimum method for assessment of quality in orthopaedic surgery.

\section{Conclusions}

The HCAHPS score, a measure of satisfaction and a determinant of Medicare quality-based reimbursement, is a measure of patient satisfaction with the hospital experience. It showed no correlation with functional outcome measures at one year after total shoulder arthroplasty. Patient satisfaction as measured by HCAHPS may not be aligned with functional outcomes valued by patients. Further consideration is warranted regarding the assessment of quality, value, and in turn reimbursements, with patient satisfaction survey results versus patient reported functional outcomes.

\section{Acknowledgments}

This work was performed at Washington University School of Medicine, St. Louis, MO, USA. 


\section{Funding: None.}

\section{Footnote}

Reporting Checklist: The authors have completed the SURGE reporting checklist. Available at http://dx.doi. org/10.21037/jhmhp-20-51

Data Sharing Statement: Available at http://dx.doi. org/10.21037/jhmhp-20-51

Conflicts of Interest: All authors have completed the ICMJE uniform disclosure form (available at http://dx.doi. org/10.21037/jhmhp-20-51). Dr. AMC reports personal fees from Arthrex, grants from Zimmer Biomet, grants from National Institute of Health, personal fees from DePuy Synthes, personal fees from Wright Medical, outside the submitted work. Dr. KY reports grants from National Institutes of Health, during the conduct of the study; personal fees from Zimmer, personal fees from Tornier, outside the submitted work; and Received $\$ 20 \mathrm{~K}$ as a monetary award related to the Ann Doner Vaughn Kappa Delta Award for the ORS/AAOS in 2014. Dr. JDK reports personal fees from Arthrex, personal fees from Shoulder Innovations, personal fees from Imascap, personal fees from Wright Medical, grants from Zimmer, grants from National Institute of Health, outside the submitted work. The other authors have no conflicts of interest to declare.

Ethical Statement: The authors are accountable for all aspects of the work in ensuring that questions related to the accuracy or integrity of any part of the work are appropriately investigated and resolved. The study was conducted in accordance with the Declaration of Helsinki (as revised in 2013). The study was approved by the Washington University in St. Louis Institutional Review Board (IRB \#: 201410148) and informed consent was taken from all patients.

Open Access Statement: This is an Open Access article distributed in accordance with the Creative Commons Attribution-NonCommercial-NoDerivs 4.0 International License (CC BY-NC-ND 4.0), which permits the noncommercial replication and distribution of the article with the strict proviso that no changes or edits are made and the original work is properly cited (including links to both the formal publication through the relevant DOI and the license). See: https://creativecommons.org/licenses/by-nc-nd/4.0/.

\section{References}

1. Center for Medicare \& Medicaid Services. Hospital Value-Based Purchasing; 2017. Available online: https:// www.cms.gov/outreach-and-education/medicarelearning-network-mln/mlnproducts/downloads/hospital_ vbpurchasing_fact_sheet_icn907664.pdf

2. Department of Health and Human Services. Medicare Program: Hospital Inpatient Value-Based Purchasing Program. Federal Registar 2011:36490-26547.

3. Center. Center for Medicare \& Medicaid Services. HCAHPS Fact Sheet; 2017. Available online: https:// hcahpsonline.org/globalassets/hcahps/facts/hcahps_fact_ sheet_november_2017.pdf

4. Glickman SW, Boulding W, Manary M, et al. Patient satisfaction and its relationship with clinical quality and inpatient mortality in acute myocardial infarction. Circ Cardiovasc Qual Outcomes 2010;3:188-95.

5. Srinivas R, Chavin KD, Baliga PK, et al. Association between patient satisfaction and outcomes in kidney transplant. Am J Med Qual 2015;30:180-5.

6. Stein SM, Day M, Karia R, et al. Patients' perceptions of care are associated with quality of hospital care: a survey of 4605 hospitals. Am J Med Qual 2015;30:382-8.

7. Tsai TC, Orav EJ, Jha AK. Patient satisfaction and quality of surgical care in US hospitals. Ann Surg 2015;261:2-8.

8. Kaye DR, Richardson CR, Ye Z, et al. Association Between Patient Satisfaction and Short-Term Outcomes After Major Cancer Surgery. Ann Surg Oncol 2017;24:3486-93.

9. Anil U, Elbuluk AM, Ziegler J, et al. Hospital Consumer Assessment of Healthcare Providers and Systems Scores Do Not Predict Outcomes After Total Hip Arthroplasty. J Arthroplasty 2018;33:337-9.e6.

10. Levin JM, Winkelman RD, Smith GA, et al. The association between the Hospital Consumer Assessment of Healthcare Providers and Systems (HCAHPS) survey and real-world clinical outcomes in lumbar spine surgery. Spine J 2017;17:1586-93.

11. Richards RR, An KN, Bigliani LU, et al. A standardized method for the assessment of shoulder function. J Shoulder Elbow Surg 1994;3:347-52.

12. Michener LA, McClure PW, Sennett BJ. American Shoulder and Elbow Surgeons Standardized Shoulder Assessment Form, patient self-report section: reliability, validity, and responsiveness. J Shoulder Elbow Surg 2002;11:587-94.

13. Wright RW, Baumgarten KM. Shoulder outcomes measures. J Am Acad Orthop Surg 2010;18:436-44. 
14. Smith MV, Calfee RP, Baumgarten KM, et al. Upper extremity-specific measures of disability and outcomes in orthopaedic surgery. J Bone Joint Surg Am 2012;94:277-85.

15. Gandhi SK, Salmon JW, Zhao SZ, et al. Psychometric evaluation of the 12-item short-form health survey (SF-

12 ) in osteoarthritis and rheumatoid arthritis clinical trials. Clin Ther 2001;23:1080-98.

16. https://www.cms.gov/Medicare/Quality-InitiativesPatient-Assessment-Instruments/HospitalQualityInits/ HospitalHCAHPS. Available online: https://www.cms. gov/Medicare/Quality-Initiatives-Patient-AssessmentInstruments/HospitalQualityInits/HospitalHCAHPS

17. Ware J Jr, Kosinski M, Keller SD. A 12-Item Short-Form Health Survey: construction of scales and preliminary tests of reliability and validity. Med Care 1996;34:220-33.

18. Day MS, Hutzler LH, Karia R, et al. Hospital-acquired conditions after orthopedic surgery do not affect patient satisfaction scores. J Healthc Qual 2014;36:33-40.

19. Kennedy GD, Tevis SE, Kent KC. Is there a relationship between patient satisfaction and favorable outcomes? Ann Surg 2014;260:592-8; discussion 598-600.

20. Sheetz KH, Waits SA, Girotti ME, et al. Patients' perspectives of care and surgical outcomes in Michigan: an analysis using the CAHPS hospital survey. Ann Surg 2014;260:5-9.

21. Iannuzzi JC, Kahn SA, Zhang L, et al. Getting satisfaction: drivers of surgical Hospital Consumer Assessment of Health care Providers and Systems survey scores. J Surg Res 2015;197:155-61.

22. Chakrabarti AK, Sheetz KH, Katariya NN, et al. Do Patient Assessments of Hospital Quality Correlate With Kidney Transplantation Surgical Outcomes? Transplant Proc 2016;48:1986-92.

23. Shirk JD, Tan HJ, Hu JC, et al. Patient experience and quality of urologic cancer surgery in US hospitals. Cancer 2016;122:2571-8

24. Joseph B, Azim A, O'Keeffe T, et al. American College of

doi: $10.21037 /$ jhmhp-20-51

Cite this article as: Wojahn RD, Atencio JM, Sefko JA, Galatz LM, Keener JD, Yamaguchi K, Chamberlain AM. Hospital satisfaction does not predict functional outcome one year after total shoulder arthroplasty. J Hosp Manag Health Policy 2021;5:36.
Surgeons Level I trauma centers outcomes do not correlate with patients' perception of hospital experience. J Trauma Acute Care Surg 2017;82:722-7.

25. Chen AL, Bain EB, Horan MP, et al. Determinants of patient satisfaction with outcome after shoulder arthroplasty. J Shoulder Elbow Surg 2007;16:25-30.

26. Peres-da-Silva A, Kleeman LT, Wellman SS, et al. What Factors Drive Inpatient Satisfaction After Knee Arthroplasty? J Arthroplasty 2017;32:1769-72.

27. Levin JM, Winkelman RD, Smith GA, et al. Impact of Preoperative Depression on Hospital Consumer Assessment of Healthcare Providers and Systems Survey Results in a Lumbar Fusion Population. Spine (Phila $\mathrm{Pa}$ 1976) 2017;42:675-81.

28. Levin JM, Winkelman RD, Tanenbaum JE, et al. Key drivers of patient satisfaction in lumbar spine surgery. J Neurosurg Spine 2018;28:586-92.

29. Maher DP, Wong W, Woo P, et al. Perioperative factors associated with HCAHPS responses of 2,758 surgical patients. Pain Med 2015;16:791-801.

30. Mistry JB, Chughtai M, Elmallah RK, et al. What Influences How Patients Rate Their Hospital After Total Hip Arthroplasty? J Arthroplasty 2016;31:2422-5.

31. Berglund DD, Law TY, Rosas S, et al. The procedure value index: a new method for quantifying value in shoulder arthroplasty. J Shoulder Elbow Surg 2019;28:335-40.

32. Menendez ME, Lawler SM, Shaker J, et al. Time-Driven Activity-Based Costing to Identify Patients Incurring High Inpatient Cost for Total Shoulder Arthroplasty. J Bone Joint Surg Am 2018;100:2050-6.

33. Menendez ME, Shaker J, Lawler SM, et al. Negative Patient-Experience Comments After Total Shoulder Arthroplasty. J Bone Joint Surg Am 2019;101:330-7.

34. Harreld K, Clark R, Downes K, et al. Correlation of subjective and objective measures before and after shoulder arthroplasty. Orthopedics 2013;36:808-14. 